\title{
Structure-property correlations in Ce-doped
}

\section{$(\mathrm{Lu}, \mathrm{Gd})_{2} \mathrm{SiO}_{5}: \mathrm{Ce}$ scintillator}

Oleg Sidletskiy ${ }^{\dagger}$, Andrei Belsky $y^{*}$ Alexander Gektin ${ }^{\dagger}$, Svetlana Neicheva ${ }^{\dagger}$, Daniil Kurtsev ${ }^{\dagger}$, Valerii Kononets $^{\dagger, t}$, Christophe Dujardin ${ }^{+}$, Kheirreddine Lebbou ${ }^{+}$, Olga Zelenskaya ${ }^{\dagger}$, Vladimir Tarasov $^{\dagger}$, Boris Grinyov ${ }^{\dagger}$

${ }^{\dagger}$ Institute for Scintillation Materials, 60 Lenin Ave., 61001 Kharkiv, Ukraine

LPCML, UMR5620 du CNRS, Université de Lyon 1, 69622 Villeurbanne cedex, France

Konstantin Belikov

SSI " Institute for Single Crystals", 60 Lenin Ave., 61001 Kharkiv, Ukraine

Full concentration range of $\mathrm{Lu}_{2 \mathrm{x}} \mathrm{Gd}_{2-2 x} \mathrm{SiO}_{5}$ (LGSO:Ce) crystals was grown by the Czochralski method. Dependence of scintillation properties on composition (x) in the range of solid solutions is established. It was determined that LGSO:Ce scintillation yield increases in the range $0.3<\mathrm{x}<$ 0.8 and reaches $29000 \mathrm{phot} / \mathrm{MeV}$ at $60 \%$ of $\mathrm{Lu}$ in the host $(\mathrm{x}=0.6)$, and energy resolution improves up to $6.7 \%$ at $662 \mathrm{KeV}$. The observed light yield increase, surprisingly high $\mathrm{Ce}^{3+}$ segregation coefficients, improvement of energy resolution and suppression of afterglow can be attributed to modification of both hot and thermalized diffusion of secondary electrons and holes induced by short-range separation in solid solution. The proposed approach can be valid for wide range of mixed scintillation crystals and provides a room for further improvement of their characteristics by isovalent substitution of host atoms. 
*Corresponding Author.

Tel.: +380-57-341-0366. Fax: +380-57-340-4474. E-mail: sidletskiy@isma.kharkov.ua 


\section{Structure-property correlations in Ce-doped $(\mathrm{Lu}, \mathrm{Gd})_{2} \mathrm{SiO}_{5}: \mathrm{Ce}$ scintillator}

Oleg Sidletskiy ${ }^{\dagger} *$, Andrei Belsky ${ }^{\dagger}$, Alexander Gektin ${ }^{\dagger}$, Svetlana Neicheva ${ }^{\dagger}$, Daniil Kurtsev ${ }^{\dagger}$, Valerii Kononets $^{\dagger,+}$, Christophe Dujardin ${ }^{*}$, Kheirreddine Lebbou ${ }^{*}$, Olga Zelenskaya ${ }^{\dagger}$, Vladimir Tarasov $^{\dagger}$, Boris Grinyov $v^{\dagger}$

${ }^{\dagger}$ Institute for Scintillation Materials, 60 Lenin Ave., 61001 Kharkiv, Ukraine

${ }^{\dagger}$ LPCML, CNRS, Université de Lyon 1, 69622 Villeurbanne cedex, France

Konstantin Belikov

SSI " Institute for Single Crystals", 60 Lenin Ave., 61001 Kharkiv, Ukraine

*Corresponding Author

Tel.: +380-57-341-0366. Fax: +380-57-340-4474.

\section{ABSTRACT}

Full concentration range of $\mathrm{Lu}_{2 x} \mathrm{Gd}_{2-2 x} \mathrm{SiO}_{5}$ (LGSO:Ce) crystals was grown by the Czochralski method. Dependence of scintillation properties on composition $(\mathrm{x})$ in the range of solid solutions is established. It was determined that LGSO:Ce scintillation yield increases in the range $0.3<\mathrm{x}<$ 0.8 and reaches 29000 phot $/ \mathrm{MeV}$ at $60 \%$ of $\mathrm{Lu}$ in the host $(\mathrm{x}=0.6)$, and energy resolution improves up to $6.7 \%$ at $662 \mathrm{KeV}$. The observed light yield increase, surprisingly high $\mathrm{Ce}^{3+}$ 
segregation coefficients, improvement of energy resolution and suppression of afterglow can be attributed to modification of both hot and thermalized diffusion of secondary electrons and holes induced by short-range separation in solid solution. The proposed approach can be valid for wide range of mixed scintillation crystals and provides a room for further improvement of their characteristics by isovalent substitution of host atoms.

\section{INTRODUCTION}

Significant amount of studies is devoted to design of mixed scintillation crystals, in which some characteristics, for example, light yield are improved, or, at least, drawbacks of mixed crystal constituents are eliminated or minimized. For example, in accordance with $[1,2]$, light yield in Ce-doped mixed lutetium-yttrium perovskite increases by around twice in comparison with $\mathrm{YAlO}_{3}: \mathrm{Ce}(\mathrm{YAP})$ and $\mathrm{LuAlO}_{3}: \mathrm{Ce}(\mathrm{LuAP})$. Similar phenomena were recently reported for $(\mathrm{LuGd})_{3}\left(\mathrm{Al}_{\mathrm{x}} \mathrm{Ga}_{1-\mathrm{x}}\right)_{5} \mathrm{O}_{12}: \mathrm{Ce}$ (LuGAGG) [3] and $\mathrm{BaBrI}: \mathrm{Eu}$ [4]. A successful example of engineering of mixed rare earth orthosilicate crystals is lutetium yttrium silicate $\operatorname{Lu}_{2 x} Y_{2-}$ ${ }_{2 x} \mathrm{SiO}_{5}: \mathrm{Ce}$ (LYSO) demonstrating improvement of energy resolution and some increase in light yield in comparison with $\mathrm{Lu}_{2} \mathrm{SiO}_{5}: \mathrm{Ce}$ (LSO) [5, 6].

Properties of scintillator at variation of solid solution composition (x) in a wide range is formed by the following processes. In solid solutions where bandgap values of its constituents differ substantially this parameter is a crucial one. The most well-known example of such solid solutions is $\mathrm{Zn}_{\mathrm{x}} \mathrm{Cd}_{1-\mathrm{x}} \mathrm{S}$ :Ag. In LuGAGG, the observed light yield increase can be attributed to the significant $(1.6 \mathrm{eV})$ bandgap change at cation substitution in solid solution [3]. In accordance 
with [4], adjusting the Ga content enables one to suppress the effect of shallow electron traps which become buried in the conduction band edge [4]. Increase of the scintillation efficiency can be also enabled by the decrease of the host band gap value, i.e., the decrease of energy for creation of one electron-hole pair. In many solid solutions the bandgap almost not changes with composition $(\Delta \mathrm{Eg} \sim 0.1 \mathrm{eV})$. In such cases the basic factor determining scintillation properties is a natural inhomogeneity of space distribution of solution forming constituents. Similarity of the observed phenomena (the light yield increase at the cation substitution ratio $x=0.3-0.7$ ) in mixed crystals with different chemical composition and space structure allows to assume more common physical mechanism. It can be related to formation of regions (clusters) enriched with one of cations leading to changes of crystalline potential on cluster boundaries and limitation of diffusion of thermalized electronic excitations. Disorder in solid solution components distribution and clusterization should modify not only the edges of conductance and valence bands. Phonon spectrum of the crystal and distribution of density of electronic states inside the bands may slow down hot carriers and, as a sequence, decrease a separation length of electronhole pair and improve scintillation yield $[7,8]$.

Previously $[9,10]$, micro-scale fluctuations of composition and interatomic distances, and formation of atomic complexes (clusters) with sizes from tens to hundreds of nanometers were observed in some metal alloys. Fluctuations of composition in the above-stated systems led to modulation of crystal potential and decrease of electron diffusion length providing the increase of electric resistance. Concerning scintillation materials, this approach was initially proposed in [2] at description of light yield trend in LuYAP:Ce solid solutions. The improvement of light yield in this system was attributed to decrease of diffusion length of thermalized uncorrelated carriers promoting their capture at $\mathrm{Ce}^{3+}$. 
The feasibility of proposed approach can be extended to other mixed scintillators. This paper deals with $\mathrm{Lu}_{\mathrm{x}} \mathrm{Gd}_{1-\mathrm{x}} \mathrm{SiO}_{5}: \mathrm{Ce}$ (LGSO:Ce) mixed crystals, which form as the result of $\mathrm{Lu} / \mathrm{Gd}$ cation substitution, that is, mixing of GSO:Ce and LSO:Ce. These materials are widely used in medicine and high-energy physics. LSO:Ce is characterized by high light yield, large effective atomic number, and fast luminescence decay. However, strong afterglow and inhomogeneity of Ce distribution along the ingots are well known drawbacks of LSO:Ce scintillator. GSO:Ce demonstrate good radiation hardness [11] and lower melting point. Unfortunately, GSO:Ce light yield is insufficient for use in modern PET scanners, and large GSO crystals are difficult to produce because of easy cleavage.

$\mathrm{Lu}_{2 \mathrm{x}} \mathrm{Gd}_{2-2 \mathrm{x}} \mathrm{SiO}_{5}$ : $\mathrm{Ce}(0<\mathrm{x}<1)$ crystals form the lattices of two types of monoclinic space symmetry [12-15]. Structure transition is observed near $x=0.2$. At $0<x<0.2$ the monoclinic $\mathrm{P} 2{ }_{1} / \mathrm{c}$ is observed, and at $0.2<x<1-$ the monoclinic $\mathrm{C} 2 / \mathrm{c}$. In the both intervals the continuous range of solid solutions is formed with smooth changes of lattice elementary cell volume (Fig. 2a). No remarkable bandgap variation change was reported for rare-earth silicates, such as $\mathrm{Lu}_{2} \mathrm{SiO}_{5}$ (LSO), $\mathrm{Gd}_{2} \mathrm{SiO}_{5}$ (GSO), however, the spread of values in different references exceeds $0.5 \mathrm{eV}$. For example, different references give the $E_{g}$ value from 6.1 to $6.6 \mathrm{eV}$ in $\mathrm{GSO}[16,17]$ and from 6 to $6.8 \mathrm{eV}$ in LSO [18-20]. Since the conductance band edge in all the range of $\mathrm{Lu}_{2 \mathrm{X}} \mathrm{Gd}_{2-2 \mathrm{x}} \mathrm{SiO}_{5}$ : $\mathrm{Ce}(0<x<1)$ crystals is formed by $5 \mathrm{~d}$ wave functions of $\mathrm{Lu}^{3+}$ and $\mathrm{Gd}^{3+}$ and the valence band is formed by $2 \mathrm{pO}$ wave functions, one may assume an insignificant bangap change $[21,22]$ with $x$.

Low light yield, similar to GSO:Ce, is inherent to LGSO:Ce at Lu concentration $<20 \%$, [14], for ex. LGSO (20\% Lu) possesses light yield approx. $15000 \mathrm{phot} / \mathrm{MeV}$ and high afterglow level up to several percents in the microsecond range [12]. The reported scintillation characteristics of LGSO:Ce with $90 \% \mathrm{Lu}$ are almost the same as those in LSO [14]. LGSO:Ce crystals with Lu 
concentration in the medium concentration range ( $30-60 \%)$ were proposed by us in [15]. They demonstrated light output around $400 \%$ relatively to $\mathrm{BGO}$, energy resolution $6.9-7.3 \%$, and afterglow level $0.02-0.1 \%$ after $5 \mathrm{~ms}$ (by 2-3 orders lower compared to LSO:Ce). Accounting for lower cost of $\mathrm{Gd}_{2} \mathrm{O}_{3}$ in comparison with $\mathrm{Lu}_{2} \mathrm{O}_{3}$ and lowering of crystallization temperature with Gd addition into the host, these crystals are good candidates, for ex., for utilization in X-ray scanners, or LSO/LYSO substitution in PET or CT.

This work deals with detailed analysis of relations between solid solution composition and scintillation characteristics in full range of $\mathrm{Lu}_{2 \mathrm{x}} \mathrm{Gd}_{2-2 \mathrm{x}} \mathrm{SiO}_{5}: \mathrm{Ce}(0<\mathrm{x}<1)$.

The potential for further improvement of LGSO:Ce characteristics by local strucuture modification can be demonstrated with $\mathrm{Ca}^{2+}$ codoped crystals. There are several papers claiming strong positive impact from codoping with divalent cations on scintillation characteristics of silicate scintillators, see for ex., $[23,24]$. As it was proposed recently [25], in LSO:Ce $\mathrm{Ca}^{2+}$ tends to occupy sixfold oxygen coordinated sites forcing out $\mathrm{Ce}^{3+}$ to sevenfold sites with higher luminescence efficiency. In this work, preliminary results on scintillation properties of LGSO:Ce,Ca scintillator are presented for the first time.

\section{EXPERIMENTAL SECTION}

\subsection{Crystal growth}

LGSO crystals with varied $\mathrm{Lu} / \mathrm{Gd}$ ratio were grown by the Czochralski method using $\mathrm{Lu}_{2} \mathrm{O}_{3}$, $\mathrm{Gd}_{2} \mathrm{O}_{3}, \mathrm{CeO}_{2}$, and $\mathrm{SiO}_{2}$ starting materials with purity not worse than $99.99 \%$ mixed at stoichiometric ratios. At growth of $\mathrm{Ca}^{2+}$ - codoped crystal, 0.05 at. $\%$ of $\mathrm{Ca}^{2+}$ was introduced into the raw material in the form of $\mathrm{CaCO}_{3}$. Crystals of $30-45 \mathrm{~mm}$ in diameter and $35-150 \mathrm{~mm}$ in length (Fig. 1) were grown in Ir crucibles by the Czochralski method with the pulling rates $1.5-$ 
$2 \mathrm{~mm} /$ hour. Growth atmosphere composition was $\mathrm{Ar}+0.3 \% \mathrm{O}_{2}$. Post-growth annealing of the ingots was carried out in oxidizing atmosphere at 1100-1200 ${ }^{\circ} \mathrm{C}$. Additionally, LSO:Ce samples were continuously annealed in oxidizing atmosphere at $1400{ }^{\circ} \mathrm{C}$ accounting for bad oxygen diffusion in them [5]. Elements with the dimensions $10 \times 10 \times 2$, and $10 \times 10 \times 0.5 \mathrm{~mm}^{3}$ annd polished 10x10 faces were cut from central part of boules for measurements of scintillation and optical characteristics.
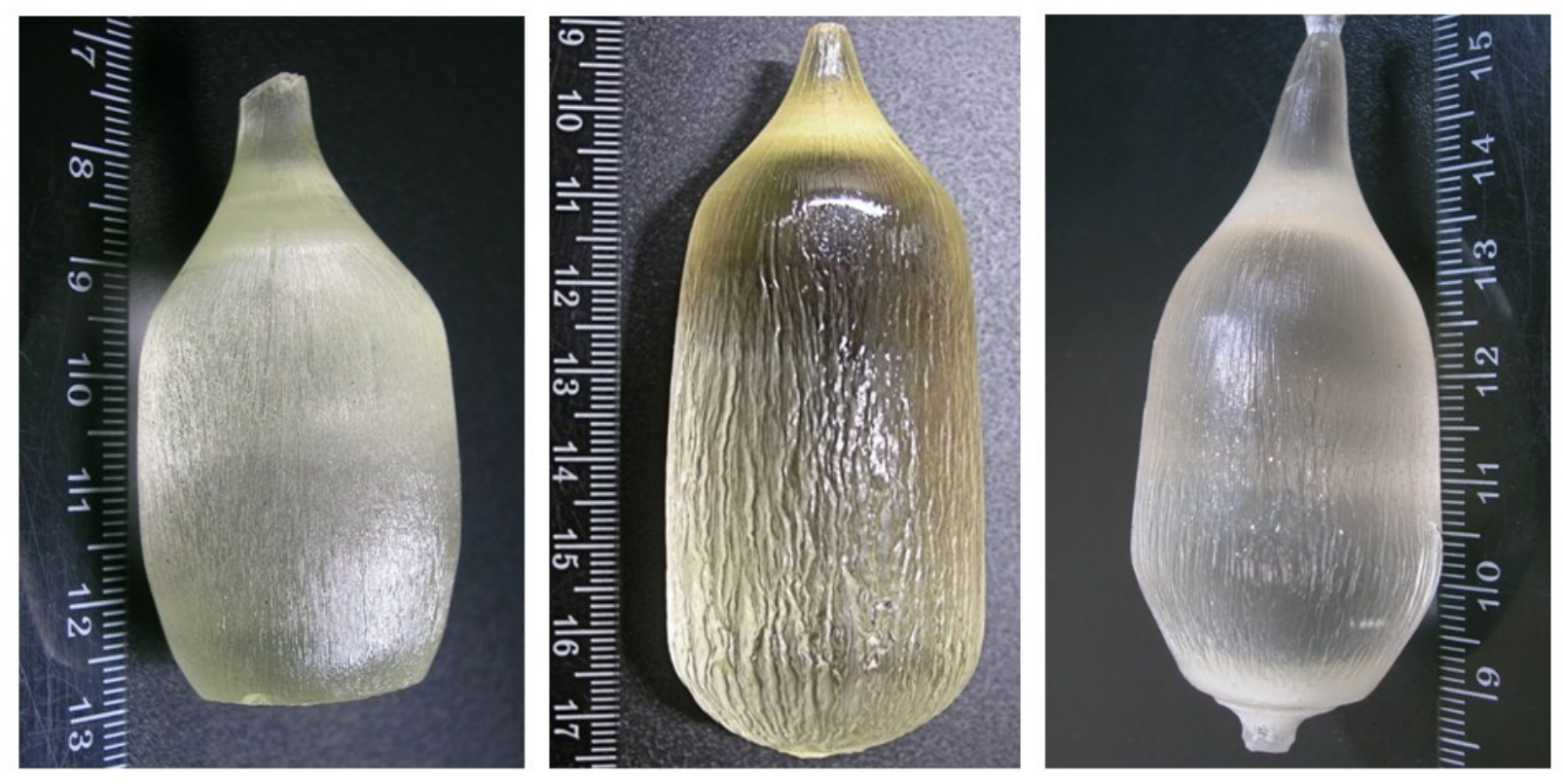

Figure 1. As-grown $\mathrm{Lu}_{\mathrm{x}} \mathrm{Gd}_{1-\mathrm{x}} \mathrm{SiO}_{5}$ :Ce crystals with $\mathrm{x}=0.5$ (left), $\mathrm{x}=0.6$ (middle), and $\mathrm{x}=0.75$ (right).

\subsection{Determination of crystal structure and cation composition.}

X-ray studies of LGSO samples were carried out using a single crystal diffractometer «Xcalibur-3» by Oxford Diffraction (MoK $\alpha$-radiation, $\lambda=0.71073 \AA$, graphite monochromator, a Sapphire-3 CCD-detector, $\omega / \theta-$ scanning in the range $2 \theta \leq 90^{\circ}$, accounting for absorption by equivalent reflections). Structure calculations were carried out using a SHELX-97 and WinGX software. Elementary cell parameters were refined by the Rietveld method, from 
diffractogrammas obtained on powders of the same crystalline samples using a Siemens D500 powder diffractometer. Results obtained by the single crystalline method were taken as initial data for refinement.

$\mathrm{Lu}$ and $\mathrm{Gd}$ content were determined by the analytical lines $\mathrm{Lu}-2615 \AA, \mathrm{Gd}-3350 \AA$ using a TRACE SCAN Advantage atomic emission spectrometer with inductively coupled plasma (ICPAES) by Thermo Jarrell Ash, USA. Lu and Gd concentrations were determined by the external standard method. Samples based on water solutions of these elements in the presence of phosphoric acid were used for calibration. The relative standard deviation of results did not exceed 0.02. Cerium concentration in LGSO crystals was determined by atomic emission spectrographic method, based on evaporation of the substance in discharge of AC arc, and registration of radiation by a DFS- 1 spectrograph. The detection limit was set to $0.001 \% \mathrm{wt}$.

Cerium segregation coefficient was determined as the ratio between Ce content in the upper part of crystal, near the symmetry axis, and Ce concentration in raw material.

\subsection{Measurement of excitation and emission characteristics.}

Excitation and emission spectra in the $230-800 \mathrm{~nm}$ range were determined using combined fluorescent lifetime and steady-state spectrometer FLS 920 (Edinburgh Instruments). Xe lamp was used for steady-state measurements, and nanosecond hydrogen-filled flashlamp was used for decay time measurements. Measurements of emission and excitation spectra in VUV were performed at Deutsches Elektronen Synchrotron (DESY, Hamburg) using the synchrotron radiation from DORIS III storage ring and facility of SUPERLUMI experiment at HASYLAB [26].

\subsection{Determination of scintillation characteristics.}

Light yield and energy resolution were measured on 10x10x2 $\mathrm{mm}^{3}$ samples with polished 10x10 faces by a pulse method described in detail in [27]. Scintillation parameters were tested 
for $662 \mathrm{keV}$ Cs-137 gamma source by a R1307 Hamamatsu PMT ran at -800V HV with linear dynode voltage divider. PMT output was connected to the charge-sensitive preamplifier BUS 295 and AMA-03F multichannel analyzer. Signal from preamplifier was transferred to custom shaping amplifier with the shaping time $2 \mu$ s. During measurements, crystals were coupled to the PMT entrance window using silicon optical compound Visilox V-788. In order to collect the whole scintillation light the crystal together with open part of PMT photocathode were covered by 3 layers of Teflon tape.

Non-proportionality of scintillator response was measured with the same $10 \times 10 \times 2 \mathrm{~mm}^{3}$ samples with polished 10x10 faces by the method similar to described in [28] (a R1307 Hamamatsu PMT was used instead of FEU-184). The following radionuclides were taken as sources of X-ray and $\gamma$-radiation: ${ }^{241} \mathrm{Am}(16.7$ and $59.5 \mathrm{KeV}),{ }^{137} \mathrm{Cs}(32.7$ and $662 \mathrm{KeV}),{ }^{133} \mathrm{Ba}$ (81 and $356 \mathrm{KeV})$.

\section{RESULTS AND DISCUSSION}

\subsection{Space structure of LGSO:Ce solid solutions.}

As one can see in Fig. 2, LGSO elementary lattice volume smoothly increases by $\sim 5 \%$ at transfer from LSO to $\mathrm{Lu}_{0.4} \mathrm{Gd}_{1.6} \mathrm{SiO}_{5}$. This lead to weak changes in crystalline field strength and to variations in positions of bangap and $5 \mathrm{dCe}$ energy levels, however, the $5 \mathrm{dCe}$ position respective to the conductance band edge does not change significantly. According to our estimations (fig.3b) the bandgap change between LSO and $\mathrm{Lu}_{0.4} \mathrm{Gd}_{1.6} \mathrm{SiO}_{5}$ (within the $\mathrm{C} 2 / \mathrm{c}$ symmetry existence range) is $0.15 \mathrm{eV}$. Since the centre of gravity of electronic shell also drifts down judging from band positions at Ce excitation spectra (рис.3a), we may propose that probability of electron capture from the conductance band by activator and ionization of $\mathrm{Ce}$ levels are constant for all the solid solution range $0.2<x<1$. 
Therefore, other factors should be considered. Substitution of $\mathrm{Lu}^{3+}$ by $\mathrm{Gd}^{3+}$ leads to loosening of the lattice and simplifies the $\mathrm{Ce}^{3+}$ incorporation into the host (Fig. 2b). It's worth to note that $k_{\text {eff }}$ dependence on host composition in Fig. 2 is not-additive with substantial deviation to larger values. While in the $\mathrm{Lu} / \mathrm{Lu}+\mathrm{Gd}$ range from 1 to $\sim 0.6$ the $k_{\text {eff }}$ increases monotonously from 0.26 to 0.55 and can be described by increase of bigger $\mathrm{Gd}^{3+}$ cation content, the further keff increase up to 0.8 is called by some another factor. This phenomenon, as well as significant dispersion of $k_{\text {eff }}$ values near the structure transition boundary is, probably, connected with inhomogeneities in crystals, namely, existence of regions (clusters) enriched with $\mathrm{Lu}^{3+}$ or $\mathrm{Gd}^{3+}$. Conditions formed at cluster boundaries may promote the incorporation of bigger quantity of activator. 


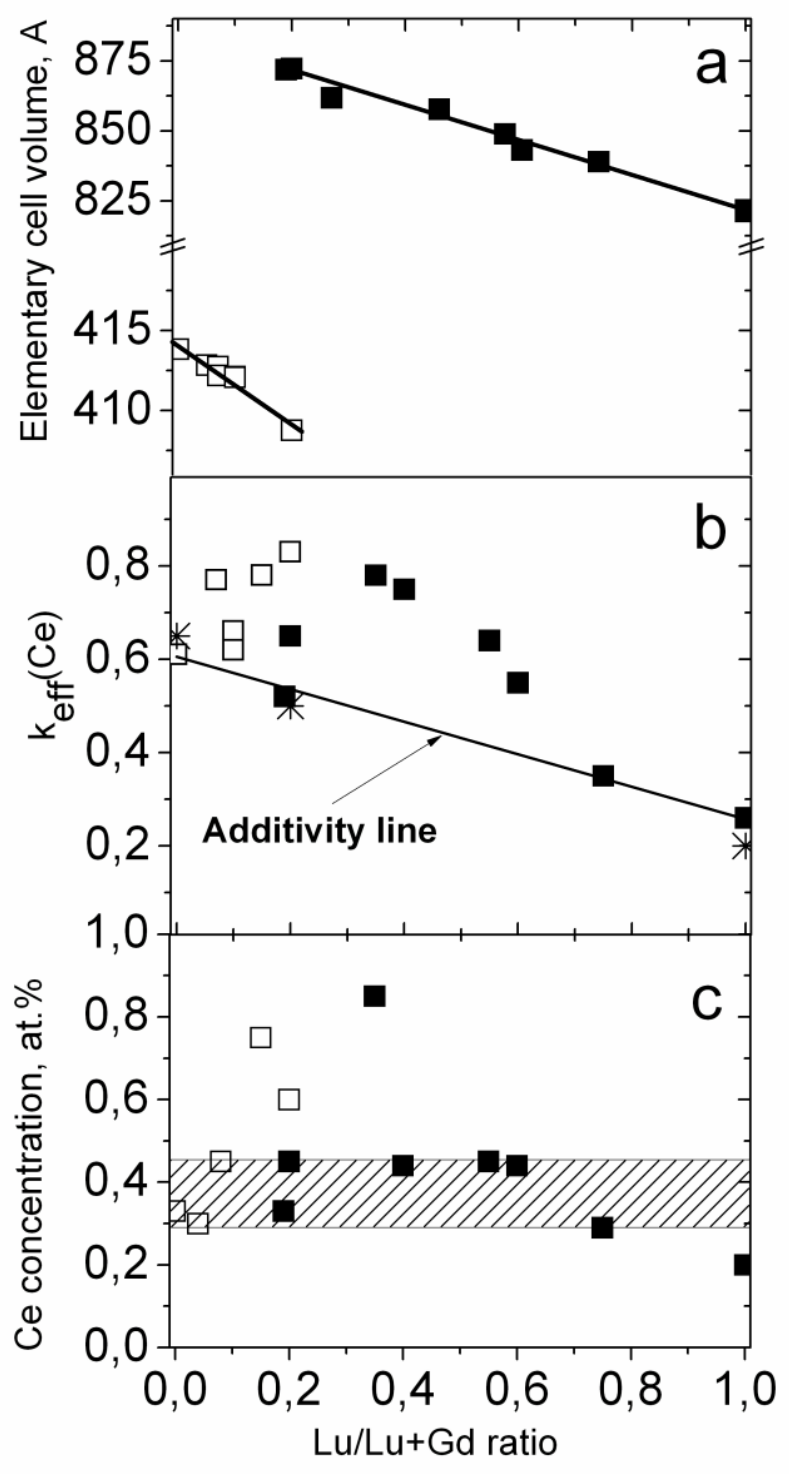

Figure 2. Elementary cell volume (a), Ce segregation coefficient (b), and Ce concentration (c) vs. LGSO host composition. The hollow symbols correspond to the $\mathrm{P} 2{ }_{1} / \mathrm{c}$ structure, and the filled symbols correspond to the C2/c structure. The asterisks at the second plot are the data from [29, 30]. The shaded area at the bottom plot corresponds to the samples with the $0.37 \pm 0.08$ at. \% Ce concentrations. 


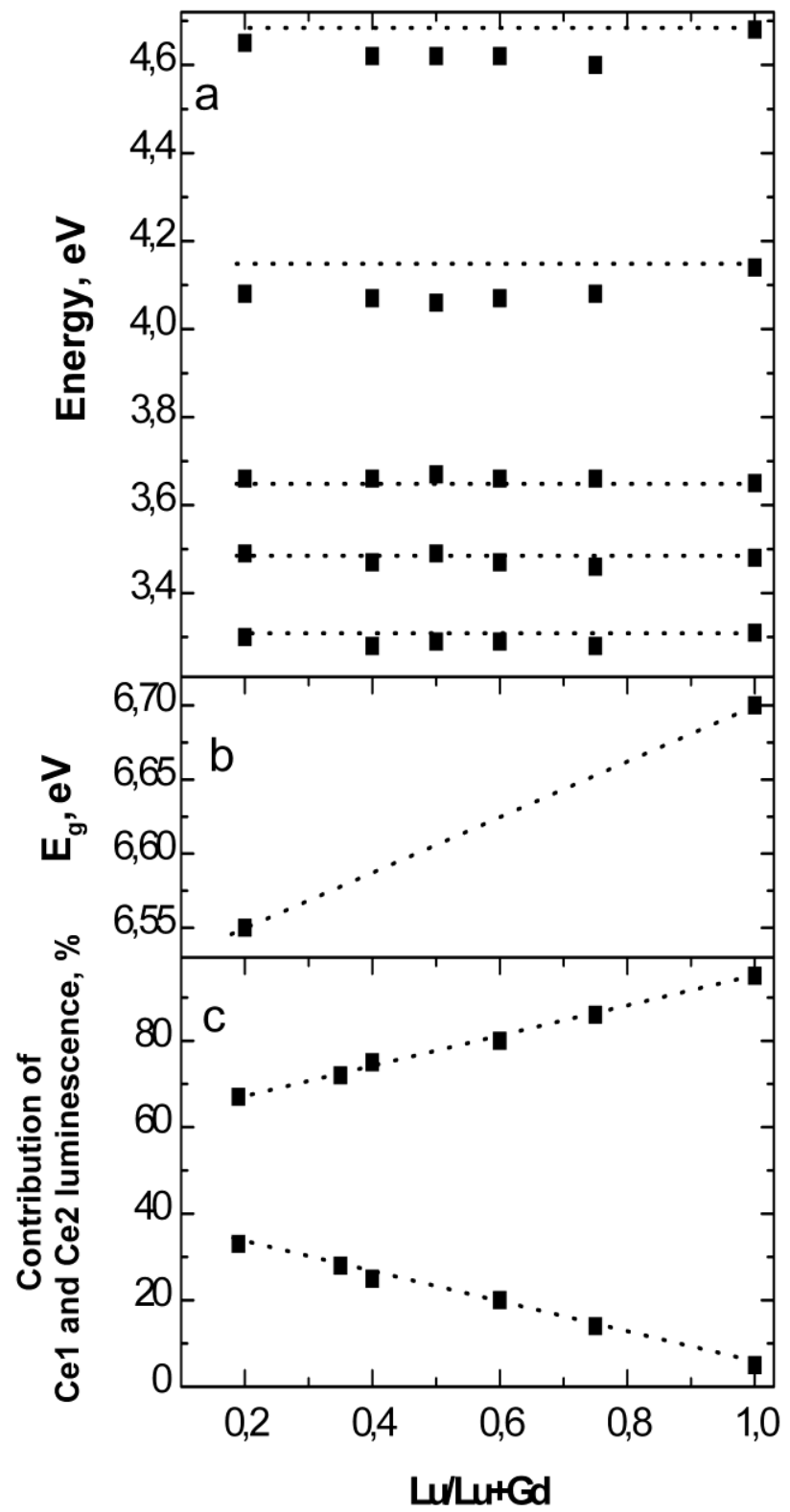

Figure 3. (a) Energies of 4f-5d transitions in obtained from excitation bands maxima of Ce1 luminescence $(\lambda \mathrm{em}=395 \mathrm{~nm})$; (b) position of the first maxima near the fundamental absorption edge in VUV excitation spectra $\left(\lambda_{\mathrm{em}}=400 \mathrm{~nm}\right)$ denoted as $E_{\mathrm{g}}$, (c) relative intensities of the Ce1 $\left(\lambda_{\mathrm{em}}=420 \mathrm{~nm}\right)$ and Ce2 $\left(\lambda_{\mathrm{em}}=510 \mathrm{~nm}\right)$ luminescence bands at X-ray excitation. The dotted lines denotes the Ce levels in LSO:Ce (a) and trend of parameters in Figs. b and c. 


\subsection{Scintillation yield and efficiency of excitation transport to activator}

Plots of basic scintillation characteristics in LGSO:Ce vs. host composition are presented in

Fig. 4. In practice, it's difficult to maintain the stable $\mathrm{Ce}^{3+}$ concentration from crystal to crystal at growth of boules with varied $\mathrm{Lu} / \mathrm{Gd}$ ratio. That is why the activator concentration in the studied LGSO:Ce crystals (see Fig. 2c) varies from 0.3 up to 0.85 at. \% (in LSO it was 0.2 at. \%). For further analysis we took the samples with the $\mathrm{C} 2 / \mathrm{c}$ structure and $\mathrm{Ce}$ concentration in the range $0.37 \pm 0.08$ at. $\%$ only (Ce concentration in LSO is $0.2 \%$ at.). In accordance with [6], this activator concentration range provides the maximal light yield in LSO:Ce.

Light yield reaches its maximum near $60 \% \mathrm{Lu}$ in the host and comprises $130 \%$ of LSO:Ce yield, while it drops by around 1.5 times as Lu concentration decreases from 60 to 20\% (Figs. 4, 5). Note that the obtained value of LSO light yield (20500 phot/MeV) is close to those published on the initial stage of LSO:Ce development $[35,36]$. Current fabrication technologies provide light yields in LSO:Ce-based crystals up to 30000 phot/MeV, or even more [37, 38]. However, in this paper, for evaluation of trend in scintillation parameters, LGSO:Ce are compared to the LSO:Ce grown in the same conditions.

With the LGSO:Ce,Ca sample with $83 \% \mathrm{Lu}$ in the host we obtained the 33700 phot/MeV light yield (Fig. 5). This is by 8500 phot/MeV more than without codoping, if to compare it with the $75 \%$ Lu sample (the closest by Lu content). However, $\mathrm{Ca}^{2+}$ codoping not influences the energy resolution of LGSO:Ce (8.1\% at $662 \mathrm{KeV})$ and afterglow $(0.46 \%$ after $5 \mathrm{~ms})$. Strong impact to scintillation yield from Ca codoping in LGSO:Ce confirms the hypothesis [ ] that $\mathrm{Ca}^{2+}$ addition leads to redistribution of $\mathrm{Ce}^{3+}$ from sixfold to sevenfold positions with higher luminescence efficiency. Note that there is a room for further light yield improvement since Lu concentration in this crystal is not quite optimal, and Ca concentration dependence have not been studied yet. 


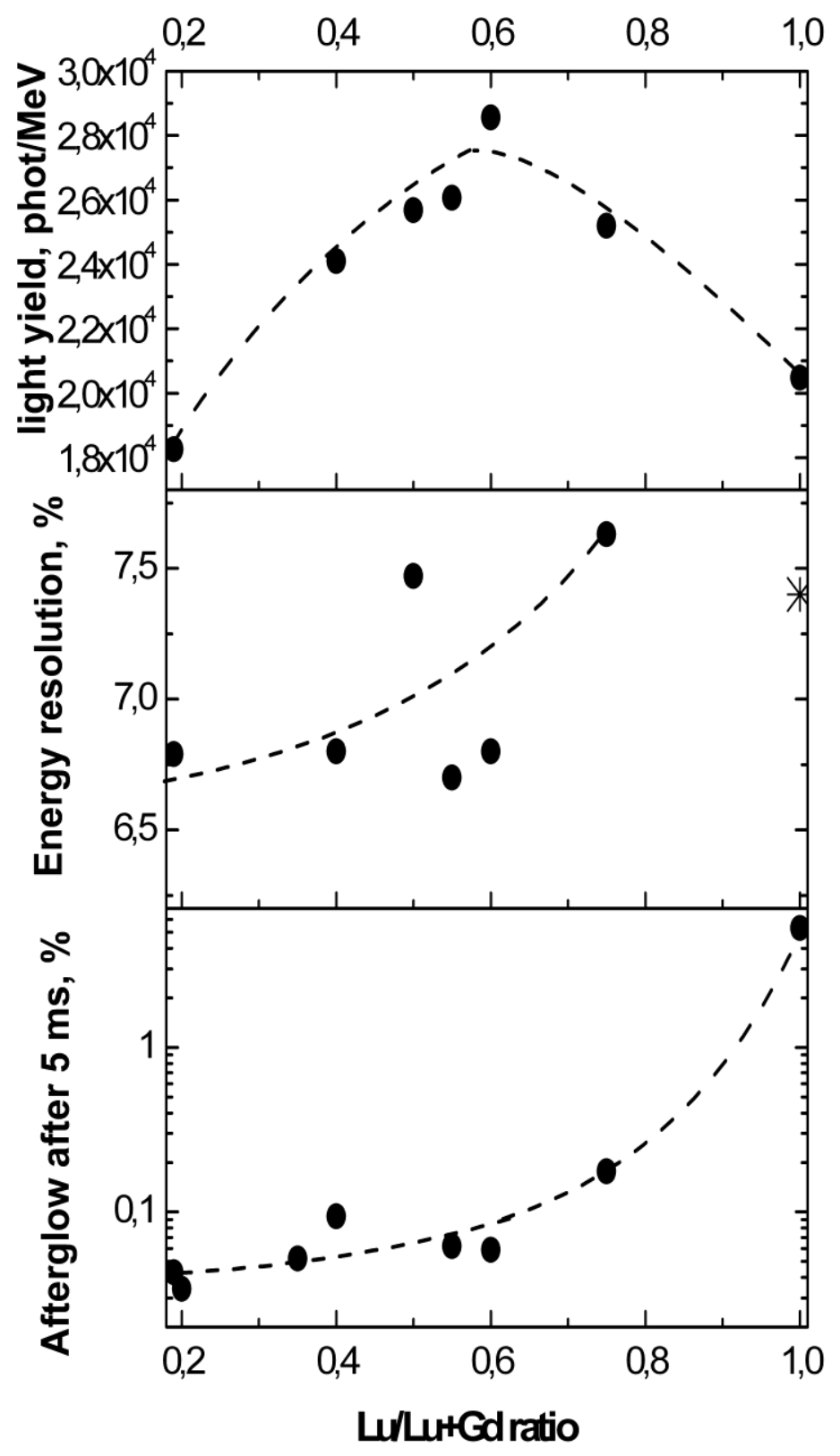

Figure 4. Light yield (a), energy resolution (b) at $662 \mathrm{KeV}$, and afterglow (c) after $5 \mathrm{~ms}$ in LGSO:Ce crystals with $\mathrm{C} 2 / \mathrm{c}$ structure vs. Lu concentration in host. The asterix in the middle figure corresponds to the best value of LSO:Ce energy resolution found in literature [39]. 


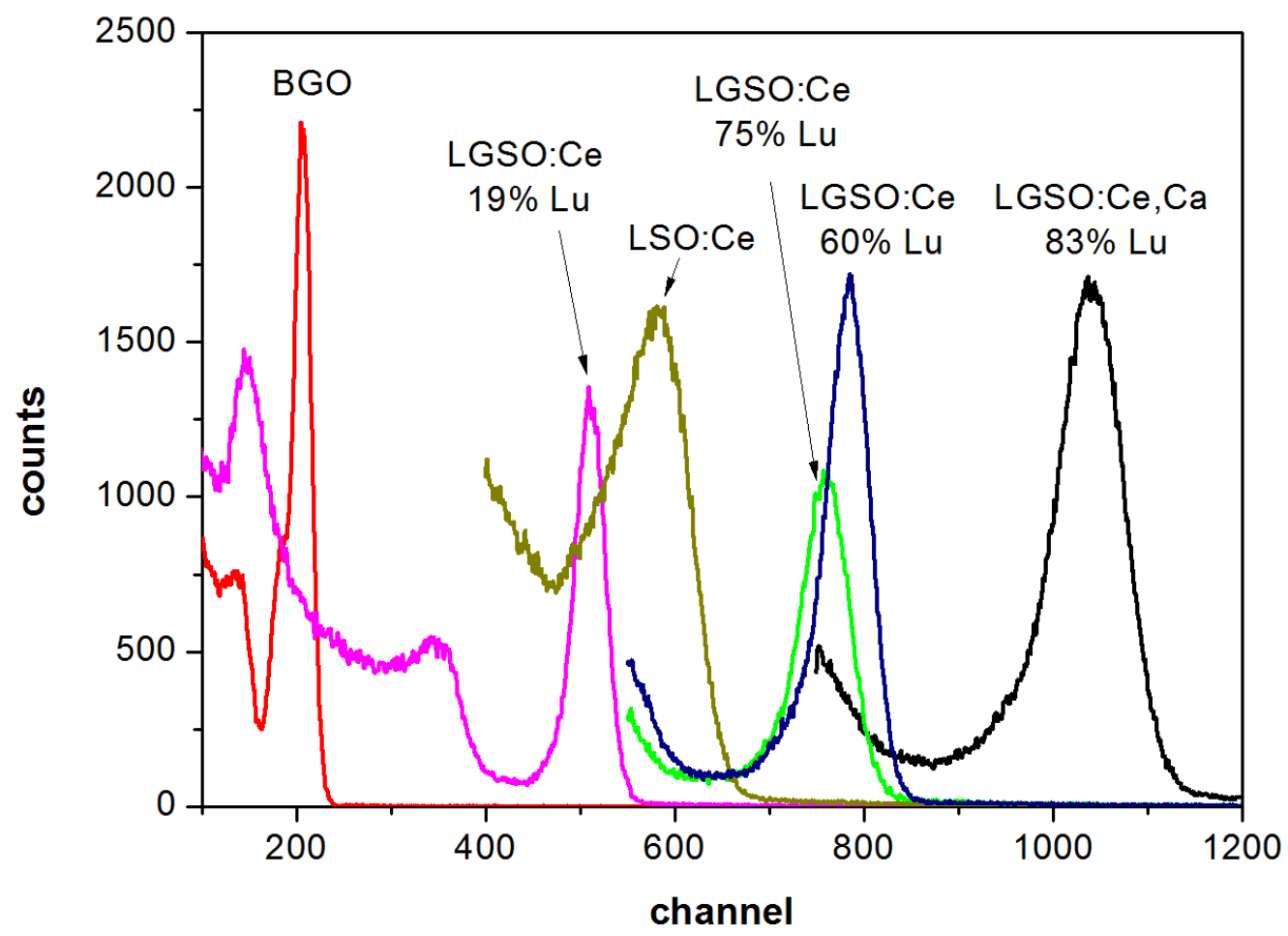

Figure 5. Pulse height spectra of some LGSO:Ce and LGSO:Ce,Ca crystals in comparison with BGO, and LSO:Ce.

The obtained trend for light yield coincides well with dependence for energy transfer efficiency to activator (Fig. 6). The hypothesis on cluster formation in the solid solution in the range around $\mathrm{x}=0.5$ accompanied by limitation of electron-hole separation and increase in light yield is confirmed by the measurements at VUV-excitation. One pair with the energy near $\mathrm{E}_{\mathrm{g}}$ is formed at excitation near the fundamental absorption edge. 2-4 e-h pairs may be formed at excitation by photons with the energies $3-5 \mathrm{E}_{\mathrm{g}}$, and the averaged energy of electrons and holes is around $1 / 2 \mathrm{E}_{\mathrm{g}}$ in respect to the conductance band bottom and valence band upper edge. During thermalization, part of them can be separated by a distance larger than cerium capture radius and will not participate in scintillation process, but lead to afterglow and other slow processes. Therefore, the 
ratio of luminescence intensities at $20-30 \mathrm{eV}\left(\sim 4 E_{g}\right)$ and 6-7 eV $\left(\sim 4 E_{g}\right)$ excitations is a characteristics of participation of secondary e-h pairs in scintillation process. This ratio is determined separately from excitation spectra of $\mathrm{Ce} 1$ and $\mathrm{Ce} 2$ centers. Note that efficiency e-h transfer to $\mathrm{Ce} 1$ excitation is about twice higher in comparison with $\mathrm{Ce} 2$. It shows that at highenergy excitation the Ce1 emission is largely dominates over the Ce2 emission. Again, the highest efficiency is obtained in the middle concentrations range.

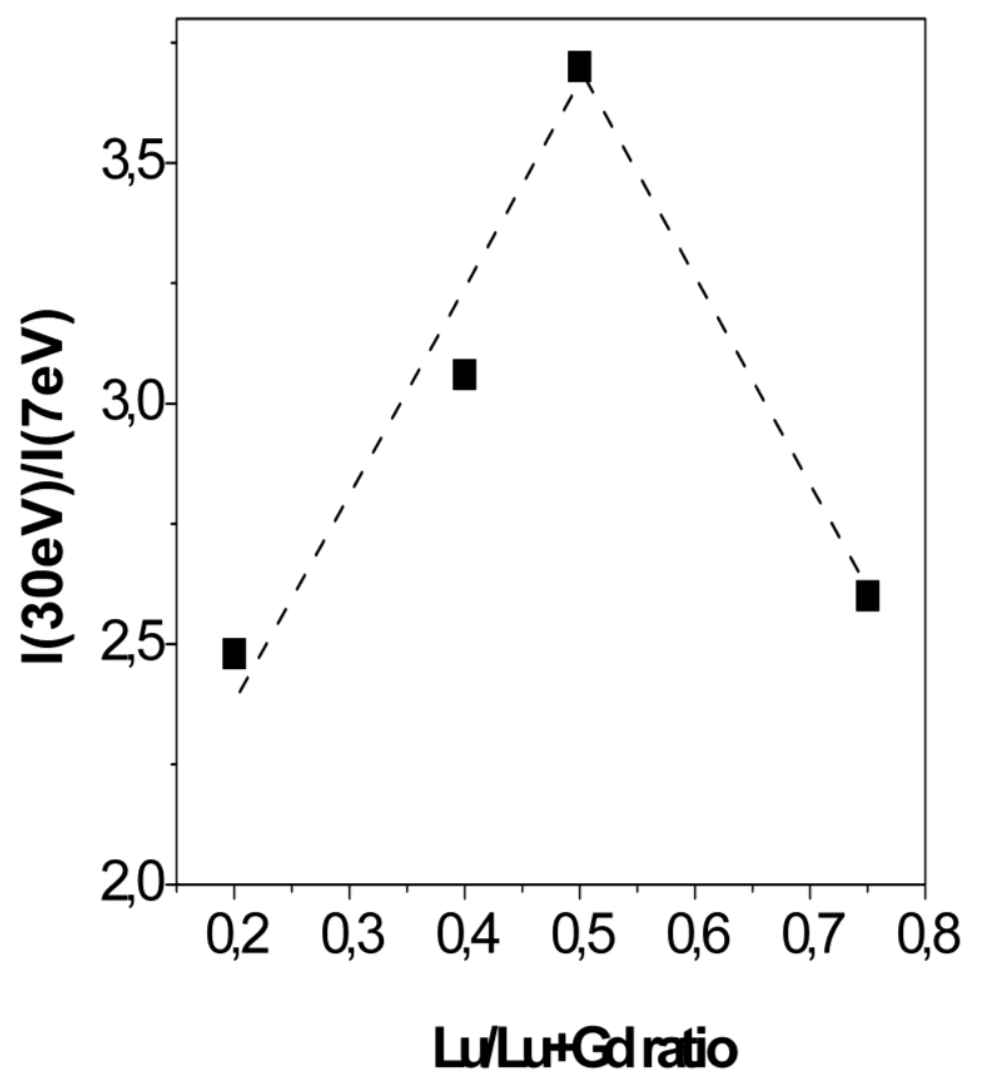

Figure 6. Ratio of excitation spectra intensities I( $30 \mathrm{eV}) / \mathrm{I}(7 \mathrm{eV})$ for $400 \mathrm{~nm}$ luminescence.

\subsection{Energy resolution and afterglow}

It was impossible to determine a certain LSO:Ce energy resolution because of the indistinct photopeak (Fig. 5). This is an evidence of crystal inhomogeneity that is inherent to this material 
[40]. The literature values for LSO range from 7.3 to $12.4 \%$ at $662 \mathrm{KeV}[39,41]$. The energy resolution between 12 and 20\% (at $511 \mathrm{KeV}$ ) was indicated in earlier publication [40]. For comparison, in Fig. 4 we plotted the best LSO reference value [39], and even in this case the energy resolution of LGSO:Ce at optimal Lu/Gd ratios is better. As one can see, energy resolution improves in the same concentration range where the strongest clusterization is expected. Thus we may suggest the correlation between energy resolution and limitation of e-h pair separation length [42]. High segregation coefficient may also influence energy resolution. We should account for the $k_{\text {eff }}(\mathrm{Ce})$ increase from 0.22 in LSO to $0.6-0.8$ in LGSO and, consequently, more homogeneous distribution of activator across the crystals. The same trendthe improvement of energy resolution up to $6 \%$ - was observed with the addition of $\mathrm{Y}^{3+}$ into LSO:Ce [6].

One should note two other factors that may influence energy resolution.

At first, energy resolution correlates with non-proportionality of scintillation response vs. energy of excitation since the energy resolution decreases as the non-proportionality increases [13]. The factors described above (e-h separation and activator distribution) are among the main parameters determining the nonproportionality. Concerning orthosilicates, non-proportionality of GSO is comparable with LGSO with $20 \% \mathrm{Lu}$ and much better than that of LSO [43]. In contrast, the LGSO with $90 \%$ of Lu showed poorer non-proportionality than that of LSO [41]. We checked the non-proportionality in LGSO with LSO-type structure in wide range of Lu concentration in host. It substantially improves at $25 \% \mathrm{Gd}$ addition into LSO, however, no substantial changes are observed at further Gd addition. Basically, this trend is similar to improvement of energy resolution with Gd addition (Fig. 4b) - the better proportionality corresponds to better energy resolution. However, note that energy resolution in LGSO is better than in both LSO with worse proportionality, and GSO with better proportionality. 
At second, it was shown [13] that, as a rule, energy resolution deteriorates in crystals exhibiting large afterglow, like LGSO, NaI(T1) or LSO, can be affected by the statistical spread of the population of the primary light pulse and that of the afterglow light. Indeed, improvement in light yield and energy resolution in LGSO:Ce is accompanied by afterglow suppression by more than 100 times in comparison with the LSO:Ce sample (Fig. 6) confirming the tendency noted in [13].

At the moment, we consider Ce distribution and separation of electron-hole pairs as basic parameters determining non-proportionality, afterglow and energy resolution in the studied solid solutions.

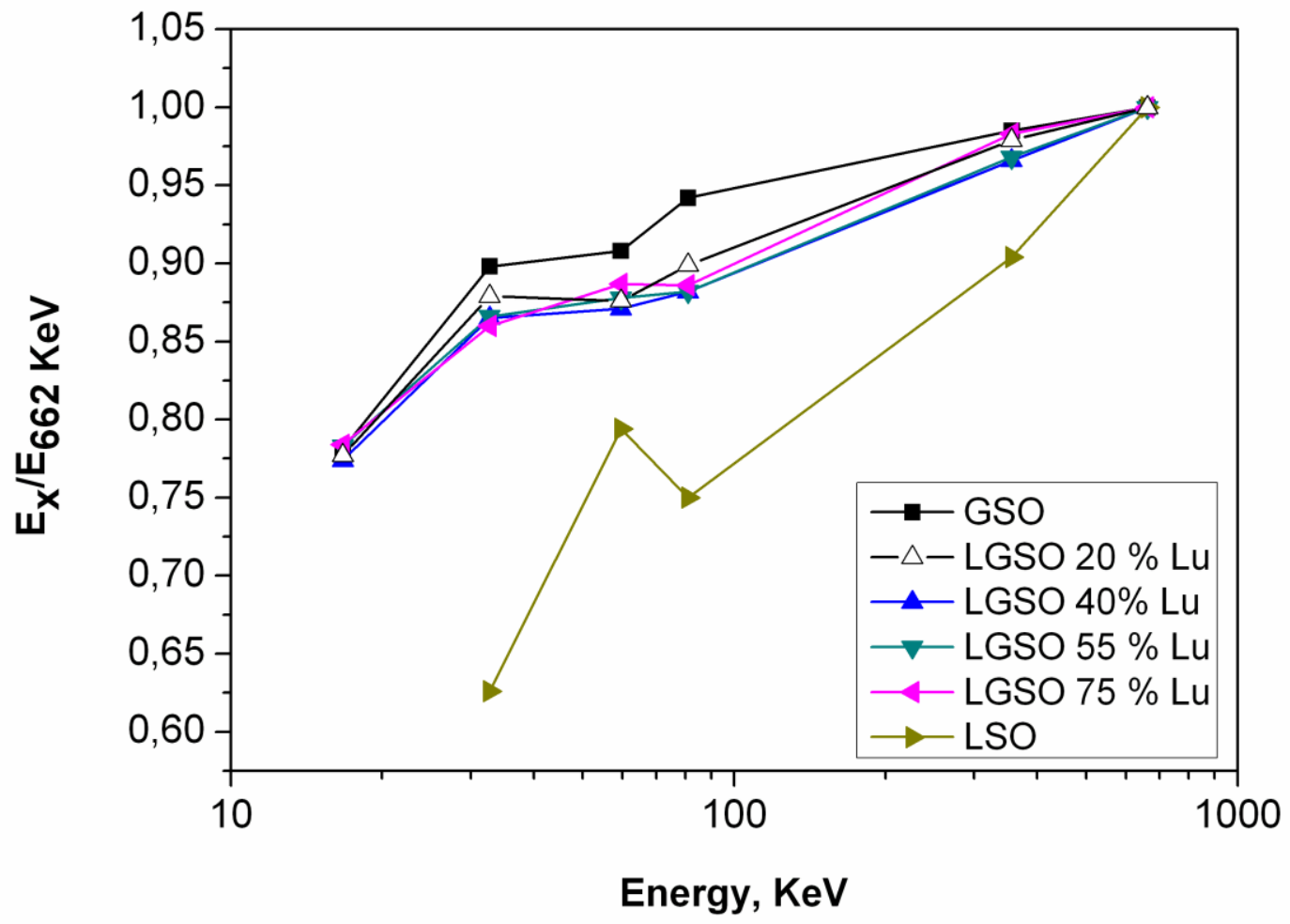

Figure 7. The non-proportionality characteristics of LGSO crystals with the different Lu contents. 


\section{CONCLUSIONS}

Correlations between local structure of LGSO:Ce crystals and their scintillation and optical properties were studied. It was established that at proper choice of $\mathrm{Lu} / \mathrm{Gd}$ ratio the LGSO:Ce scintillation yield reaches 29000 phot/MeV ( $130 \%$ compared to LSO:Ce grown in the same conditions). At the same time, energy resolution improves up to $6.7 \%$ at $662 \mathrm{KeV}$, and afterglow decreases by 2 orders of magnitude. With $\mathrm{Ca}^{2+}$ codpoing, the 33700 phot/MeV light yield was achieved. Thus, LGSO crystals possess a very attractive combination of characteristics for practical applications in medicine, security systems, high-energy physics.

The approach is proposed which describes the observed improvement in scintillation characteristics by formation of Lu- and Gd-enriched regions in crystals. This short-range separation in solid solution may lead to formation of potential barriers limiting the e-h separation length and increasing the probability of carrier capture on $\mathrm{Ce}^{3+}$ ions and subsequent $5 \mathrm{~d}-4 \mathrm{f}$ radiative transitions. This model can be confirmed by direct study of local crystal structure in different solid solution crystals (EXAFS, or neutron scattering, etc). At the moment, several indirect evidences of non-homogeneous distribution of $\mathrm{Lu}$ and $\mathrm{Gd}$ in the host is obtained: (i) very similar increase of light yield in LGSO and other solid solutions with different composition and structure; (ii) non-additive improvement of Ce segregation coefficient; (iii) increase of e-h transfer efficiency to activator.

The proposed mechanism can be valid for a wide range of mixed scintillation crystals and provides a room for further improvement of scintillator characteristics in them.

\section{AUTHOR INFORMATION}


*Corresponding Author. Tel.: +380-57-341-0366. Fax: +380-57-340-4474. E-mail: sidletskiy@isma.kharkov.ua

\section{ACKNOWLEDGMENT}

The work was partially supported by the Ukrainian-French scientific project N23997of NAS of Ukraine and CNRS "Structure, electronic and scintillation properties of mixed crystals based on silicates of lutetium and gadolinium", Project FP7-INCO-2011-6 "Strengthening Ukraine and EU research cooperation in the field of Material Sciences" ("SUCCESS"), and CNRS scholarship N722792L for a short-term research stay in France.

\section{REFERENCES}

(1) Petrosyan, A.G.; Ovanesyan, K.L.; Shirinyan, G.O.; et al. J. Cryst. Growth 2000, 211, 252256.

(2) Belsky, A.N.; Auffray, E.; Lecoq, P.; et al. IEEE Trans. Nucl. Sci. 2001, 48, 1095-1100.

(3) Kamada, K.; Endo, T.; Tsutumi, K.; et al. Cryst. Growth Des. 2011, 11, 4484-4490.

(4) Fasoli, M., Vedda, A., Nikl, M et al., Phys. Rev. 2011. B 84, 081102(R).

(5) Chai. B., Method of Enhancing performance of cerium doped lutetium yttrium orthosilicate crystals and crystals produced thereby. U.S. Patent 7,166,845 B1, January 23, 2007. United States Patent and Trademark Office Web site. http://patft.uspto.gov/netacgi/nph- 
$\underline{\text { Parser} ? \text { Sect } 1=\text { PTO } 1 \& \text { Sect } 2=\text { HITOFF } \& d=\text { PALL } \& p=1 \& u=\% 2 \text { Fnetahtml } \% 2 F P T O ~} \% 2$ Fsrchnum.h $\underline{\mathrm{tm} \& \mathrm{r}=1 \& \mathrm{f}=\mathrm{G} \& \mathrm{l}=50 \& \mathrm{~s} 1=7,166,845 . \mathrm{PN} . \& \mathrm{OS}=\mathrm{PN} / 7,166,845 \& \mathrm{RS}=\mathrm{PN} / 7,166,845}$ (accessed July 10, 2012).

(6) Chen, J.; Zhang, L.; Zhu, R.-Y.; IEEE Trans. Nucl. Sci. 2005, 52, 3133-3140.

(7) Vasil'ev, A. N.; Mikhailin, V. V., Izv. Akad. Nauk, Ser. Fiz. 1986, 50(3), 113-116.

(8) Glukhov, R. A.; Vasil'ev, A. N., Radiat. Eff. Defects Solids 1995,135, 813-817.

(9) Iveronova, V. I.; Katsnelson, A. A. Short-range Order in Solid Solutions; Nauka: Moscow, 1977; 256 p.

(10) Batyrev, I.G.; Leiro J.A.; Nikiforova, L.A.; Katsnelson, A.A. J. Phys. Chem. Solids 1993, $54,779-784$.

(11) Kobayashi, M.; Ieiri, M.; Kondo, K.; et al. Nucl. Instr. Meth. Phys. Res. Sect. A 1993, 330, $115-120$

(12) Loutts, G.B.; Zagumenni, A.I.; Lavrishichev, S.V.; et al. J.Cryst. Growth 1997, 174, 331336.

(13) Moszyński M.; Nassalski, A.; Czarnacki, W.; et al. IEEE Trans. Nucl. Sci. Symp. Conf. Rec. 2006, N37, 1493-1499.

(14) Shimizu, S.; Pepin, C.M.; Bergeron, M.; et al. IEEE Trans.Nucl. Sci. 2010, 57(1), 55-61.

(15) Sidletskiy, O.; Bondar, V.; Grinyov, B.; et al. J. Cryst. Growth 2010, 312, 601-606.

(16) Yang, J.-K.; Park, H.-H.; Appl. Surf. Sci. 2005, 244, 293-296. 
(17) Chen, Y.; Liu, B.; Shi, C.; et al. J. Phys.: Condens. Matter 2005, 17, 1217-1224.

(18) Znamenskii, N.; Manykin, A.; Petrenko, E.; et al. J. Exp. Theor. Phys. 2004, 99, 386-393.

(19) Melcher, C. L.; Schweitzer, J. S.; IEEE Trans. Nucl. Sci. 1992, 39, 502-505;

(20) Vedda, A.; Nikl, M.; Fasoli, M.; et al. Phys. Rev. 2008, B 78, 195123.

(21) Dorenbos, P, J. Phys.: Condens. Matter 2003, 15, 2645

(22) Thiel, C.W.; Gruguel, H; Wu, H.; et al. Phys. Rev. B 2001, 64, 085107

(23) Zavartsev Yu. D.; Koutovoi S.A.; Zagumennyi A.I. Journal of Crystal Growth 2005, 275 e2167-e2171.

(24) Rothfuss, H.; Melcher, Ch.; Eriksson, L.; et al. IEEE Trans. Nucl. Sci., 2009, 56(3), 958961.

(25) Yang K., Melcher Ch., Koschan, M.; and Zhuravleva, M.; IEEE Trans. Nucl. Sci., 2011, 58(3), 1394-1399.

(26) Zimmerer, G. Nucl. Instr. Meth. Phys. Res. A 1991, 308, 178-186.

(27) Sysoeva, E.; Tarasov, V.; Zelenskaya, O.; Nucl. Instr. and Meth. Phys. Res. A 2002, 486, $67-73$.

(28) Sysoeva, E.P.; Zelenskaya O.V.; Sysoeva, E.V.; IEEE Trans. Nucl. Sci. 1996, 43(3), 12821283.

(29) Brandle, C.D.; Valentino, A.J.; Berkstresser, G.W.; J. Crystal Growth 1986, 79, 308-315. 
(30) Usui, T.; Yamamoto, H.; Yokoyama, Y.; et al. IEEE Trans Nucl Sci. 2007, N24-206, 1412-1416.

(31) Shannon, R.D. Acta Crystallogr, 1976, A32, 751-767.

(32) Suzuki, H.; Tombrello, T. A.; Melcher, C. L.; et al. IEEE Trans. Nucl. Sci. 1993, 40(4), 380-383.

(33) Liu, B.; Shi, Ch.; Yin ,M.; et al.; J. Lumin. 2006, 117, 129-134.

(34) Usui T.; Shimizu, S.; Shimura N.; IEEE Nucl. Sci. Symp. Conf. Rec., 2006, N30-142, 1166-1169.

(35) Melcher, C.L.; Sweitzer, J.S.; IEEE Trans. Nucl. Sci., 1992, 39(4), 502-505.

(36) Dorenbos, P.; van Eijk, C.W.E.; Bos, A.J.J.; and Melcher, C.L.; J. Phys.: Condens. Matter, 1994, 6, 41674180.

(37) Lawrence Berkeley National Laboratory. Table of Scintillation Properties. http://scintillator.lbl.gov/ (accessed July 9, 2012).

(38) Grodzicka, M.; Moszynski, M.; Szczesniak, T; et al. Nucl. Instr. Meth., 2011, A 652, 226230.

(39) Kapusta, M.; Moszynski, M.; Balcerzyk, M.; et. al, IEEE Trans. Nucl. Sci, 2000, 47, 13411345.

(40) Melcher C.L. Manufacturing a cerium-doped lutetium oxyorthosilicate boule. Patent WO 2002068733, September 6, 2002. World intellectual Property Organization Website. http://patentscope.wipo.int/search/en/detailPdf.jsf?ia=US2002006013\&docIdPdf $=090063618008$ 
$\underline{\text { e37c\&name }=(\text { WO2002068733) }) \text { MANUFACTURING A CERIUM-DOPED LUTETIUM }}$ OXYORTHOSILICATE SCINTILLATOR BOULE\&woNum=WO2002068733\&prevRecNum

$=1 \&$ nextRecNum=2\&recNum=1\&queryString=IN\%3Amelcher $+\&$ office=\&sortOption=\&prevFil $\underline{\text { ter }=\& \max R e c}=1($ accessed July 10, 2012)

(41) Balcerzyk, M., Moszyński, M., Kapusta, M.; et al. IEEE Trans. Nucl. Sci., 2000, 47, 13191323.

(42) Gektin

(43) Moszynski, M.; Nassalski, A.; Czarnacki, W.; et al. IEEE Trans. Nucl. Sci., 2007,54, 725731. 
For Table of Contents Use Only
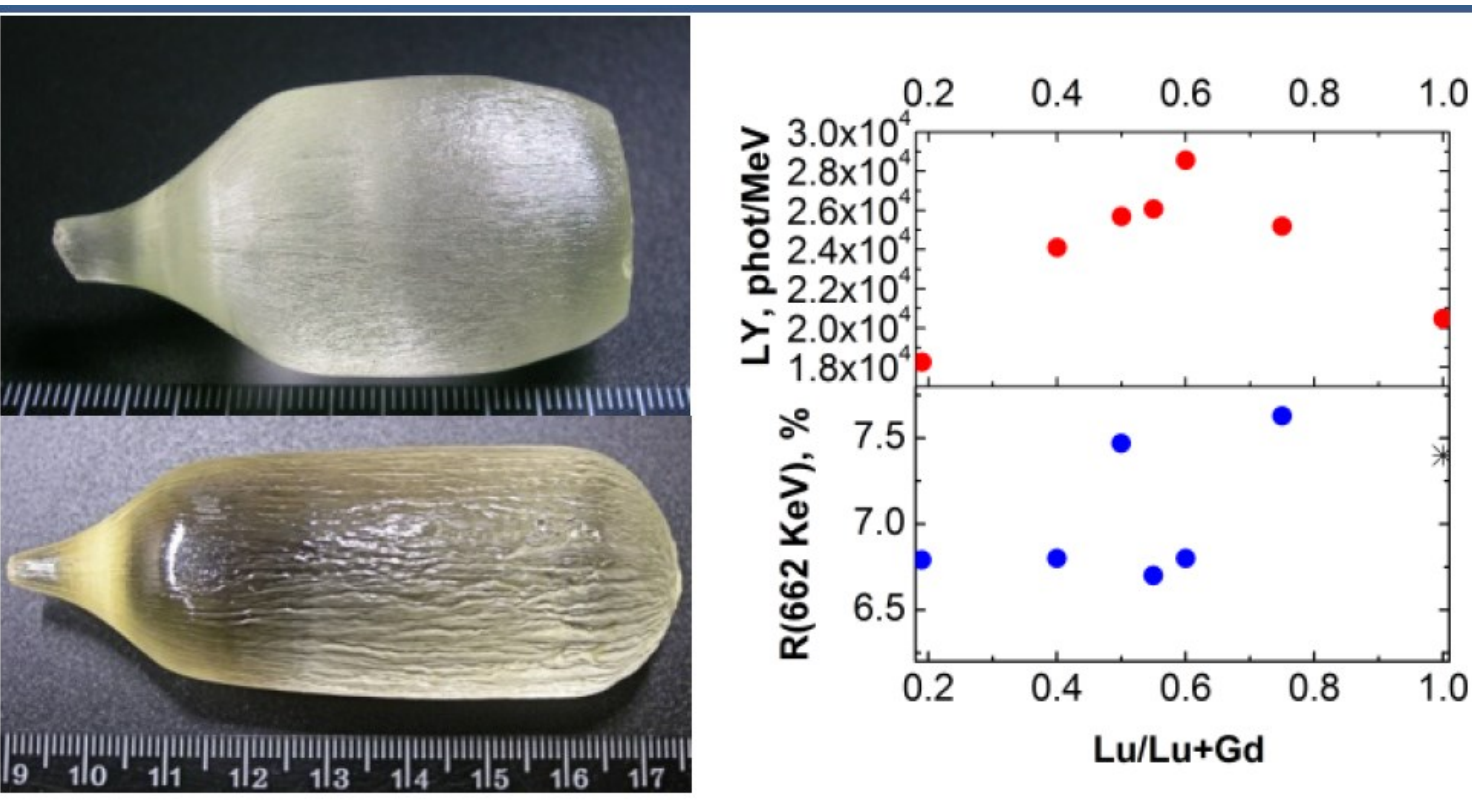

SYNOPSIS.

- Scintillator crystals based on $\mathrm{Lu}_{\mathrm{x}} \mathrm{Gd}_{1-\mathrm{x}} \mathrm{SiO}_{5}$ (LGSO) with light yield up to 33600 phot/MeV are grown by the Czochralski method.

- Correlations between local structure and scintillation characteristics in LGSO:Ce are discussed.

- A mechanism of light yield improvement by short-range separation in solid solution is proposed. 